\title{
Wertschöpfung verlangt Wertschätzung
}

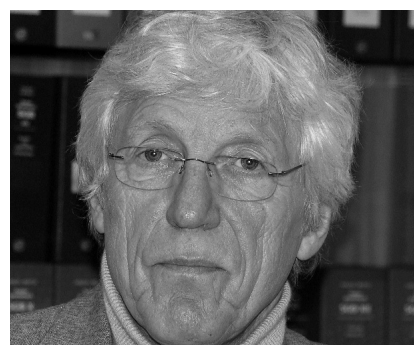

VON WOLFGANG HOFFMANN Wolfgang Hoffmann (59) ist Dozent an der Bundesakademie für Kirche und Diakonie gGmbH und an der Führungsakademie für Kirche und Diakonie gAG. Er ist dort u. a. für die Themen Management in sozialen Organisationen und Personalentwicklung verantwortlich.

E-Mail hoffmann@bundesakademie-kd.de

\author{
Die Freie Wohlfahrtspflege hat ein gutes Ansehen in der \\ Öffentlichkeit - aber nicht unbedingt als Beschäfti- \\ gungssektor. Zu viele Organisationen und Unterneh- \\ men haben sich in den letzten Jahren nicht als attrakti- \\ ve Arbeitgeber empfohlen.
}

Kaum ein Thema beschäftigt Unternehmen in Deutschland derzeit mehr als der »Fachkräftemangel «. Und kaum eine andere Branche als die Organisationen der Sozialwirtschaft ist bei der Erbringung ihrer Leistungen so sehr auf gut ausgebildete, leistungsbereite Mitarbeitende angewiesen. Aber stimmt der Eindruck, dass es gerade diese Branche ist, die sich noch immer kaum bemüht, für Mitarbeitende attraktiv zu sein? Nie war die folgende Frage aktueller als jetzt: »Jeden Abend verlassen 80 Prozent des Kapitals Ihrer Firma. Was tun sie, damit es morgen zurückkehrt? "

Noch zu viele Unternehmen sehen in ihren Mitarbeitenden nicht das entscheidende »Kapital «, mit dem man Erfolge erzielen kann, sondern eher den Kostenfaktor, den es zu minimieren gilt. Nur: Wer heute die Mitarbeitenden noch vorwiegend als » Kostenfaktor « behandelt, darf sich morgen nicht wundern, wenn in diesem Unternehmen keiner mehr arbeiten will.

\section{Was soziale Unternehmen tun können}

Ein Unternehmen attraktiv für Mitarbeitende zu gestalten, ist nicht eine Frage des Geldes. Materielle Leistungen haben eine eher untergeordnete Bedeutung. Arbeitnehmerinnen und Arbeitnehmer sind immaterielle Werte wie Wertschätzung, Gestaltungsmöglichkeiten, Beteiligung bei Entwicklungs- und Entscheidungsprozessen und ein Respekt vor der geleisteten Arbeit viel wichtiger.

Damit die »Arbeitgeberattraktivität « ihre Wirkung voll entfalten kann, müssen Managementstrukturen nach innen eine konsequente Mitarbeitendenorientierung markieren und die Arbeitgebermarke nach außen eine gesellschaftliche Anerkennung für den Dienst am Menschen fördern. Einer Kultur der Wertschätzung muss in einem abgestimmten, transparenten System aus Instrumenten der Unternehmensführung und Mitarbeitendengewinnung die Wertschöpfung folgen!

Sechs Instrumente der Unternehmensführung und Mitarbeitendengewinnung sind entscheidend dafür, wie attraktiv ein Unternehmen für Mitarbeitende ist.

\section{Erster Erfolgsfaktor: Das Management-Modell}

Ein klar formuliertes ManagementModell, in dem die Ressourcen der Mitarbeitenden in Entwicklungs- und Entscheidungsprozessen einbezogen werden, wird als Grundlage der Mitarbeitendenorientierung wahrgenommen. Dabei geht es darum, die Innovationskraft der Mitarbeitenden mit ihren vielfältigen Begabungen, Fachlichkeiten, Gestaltungswillen und Kulturen als Potentiale für das Unternehmen zu nutzen.

Das Modell »Management by participation « (Führen durch Teilhabe und Beteiligung) sieht eine starke Beteili- 
gung der Mitarbeitenden bei der Zielentwicklung, Entscheidungsfindung und Aufgabenerfüllung vor. So wird die Identifikation mit den Unternehmenszielen erhöht. Mitarbeitende und Unternehmensleitung sind Partner in der Leistungserbringung. Diese Kultur der Partnerschaft macht ein Unternehmen attraktiv für potentielle Bewerberinnen und Bewerber.

\section{Zweiter Erfolgsfaktor: Die Führungskonzeption}

Aus dem Managementmodell werden in einer Führungskonzeption Regeln für das Leitungshandeln verbindlich abgeleitet. Für die meisten Handlungsfelder sozialwirtschaftlicher Unternehmen geben Konzepte den Mitarbeitenden Handlungssicherheit (pädagogische Konzepte, Rahmenfinanzpläne, Pflegevorschriften, Marketingüberlegungen etc.). Nur das Leitungshandeln wird weitgehend unorganisiert an die verschiedenen Führungsebenen delegiert.

Jede Führungskraft steht täglich vor der Aufgabe, den Balanceakt zwischen Zielen und Vorgaben des Unterneh- mens einerseits, und sozialen und partizipativen Aspekten andererseits zu meistern. Dabei bleiben zu oft die legitimen Interessen der Mitarbeitenden nach einer hohen Betreuungsqualität und angemessenen Arbeitsbedingungen auf der Strecke. Eine Führungskonzeption muss einen verbindlichen Rahmen für die Führungsaktivitäten im gesamten Unternehmen definieren. Folgende Aussagen in einer Führungskonzeption sollten die Qualität des Leitungshandelns optimieren:

- Definition von Leitungshandeln (z. B. hierarchisches Leiten von oben oder partizipatives Steuern von Prozessen)

- Stellung der Mitarbeitenden (z. B. Mitarbeitende als »funktional dienende Kräfte « oder als »innovatives Kapital «)

- Wie werden Ziele entwickelt und Entscheidungen getroffen? (Z. B. durch Vorgabe der Geschäftsleitung oder unter Einbeziehung der Mitarbeitenden, die an der Erreichung der Ziele und der späteren Umsetzung der Entscheidung mitwirken sollen)
- Wie werden die innovativen Kräfte der Mitarbeitenden gefördert und gefordert? (Z. B. durch eine projektorientierte Übertragung von Verantwortung für Entwicklungsprozesse)

- Welche Kritikkultur wird erwartet? (Z. B. welche Bedeutung hat Kritik als Steuerungsinstrument, welchen Stellenwert haben Feedback-Runden, wie sind Rückmeldungen hierarchieübergreifend geregelt?)

- Wie wir mit Konflikten umgegangen, welche Konfliktkultur ist gewünscht? (Z. B.: Werden Konflikte in einer Konfliktvermeidungskultur und einer Konfliktverdrängungskultur vermieden oder als Chance verstanden?)

- Bedeutung der Personalentwicklung? (Z. B. als eine Störung im Betriebsablauf oder als fester Bestandteil in der Unternehmensführung zur Optimierung der Ressource Mitarbeitende?)

- Stellenwert eines betriebliches Gesundheitswesen? (Z. B. beschränkt auf die »Unfallverhütungsvorschriften " oder versteht sich als eine aktive Förderung der Gesundheit der Mit-

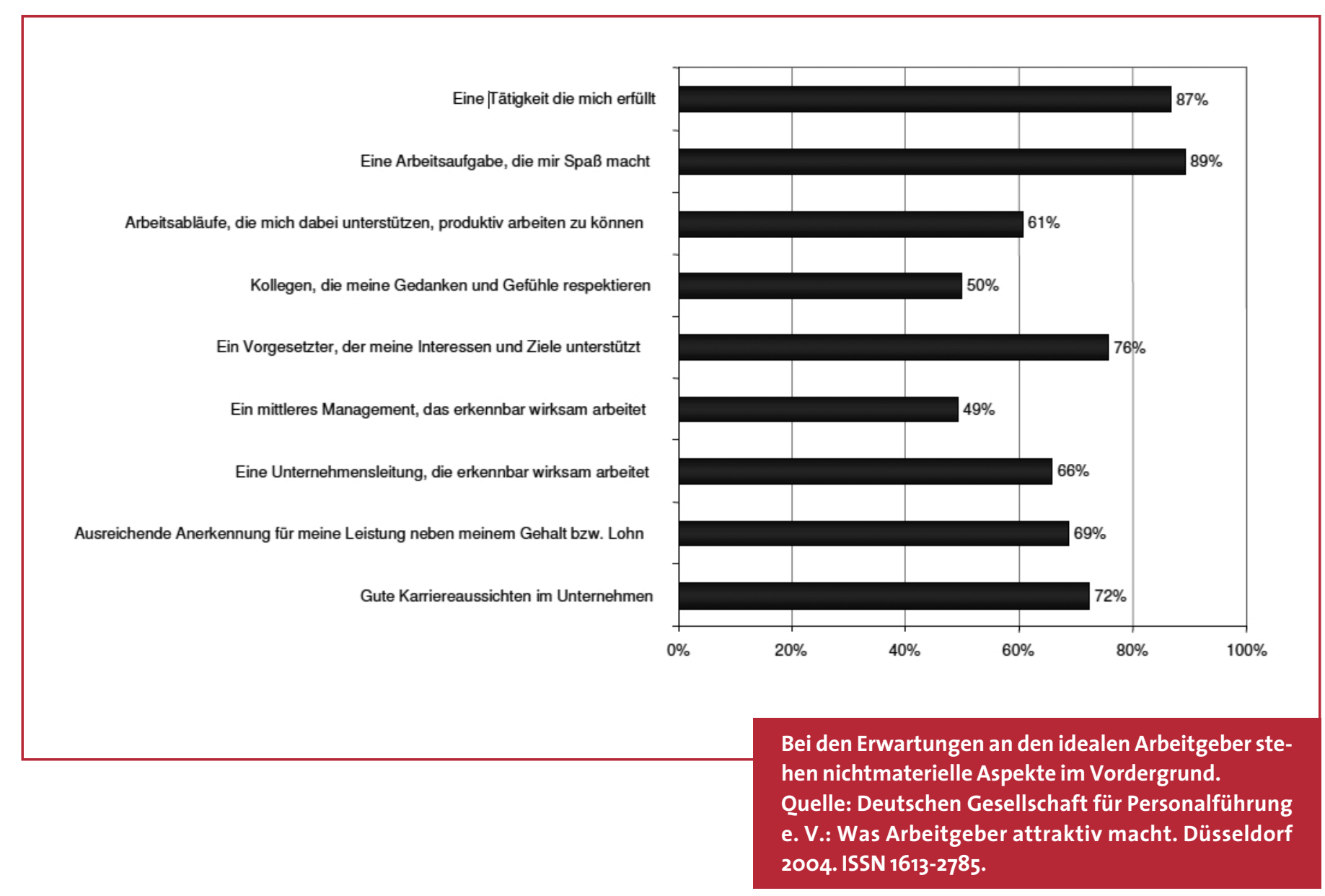


arbeitenden zum Erhalt ihrer Leistungsfähigkeit?)

Interessierte Bewerberinnen und Bewerber sollen wissen, wie mit ihnen im Unternehmen umgegangen wird. Eine Führungskonzeption schafft Transparenz und Vertrauen! um so positiver fällt die Bilanz aus. So ziehen quasi zwangsläufig engagierte Mitarbeiterinnen und Mitarbeiter den zufriedenen Kunden und damit das bessere Resultat nach sich. Der Ressource Mitarbeitende kommt damit zunehmend eine wirtschaftliche Schlüsselrolle zu.

\section{"50-jährige Mitarbeitende haben heute noch 17 Berufsjahre vor sich"}

\section{Dritter Erfolgsfaktor: Die Personalentwicklung}

Der weitaus größte Anteil der Gesamtkosten eines dienstleistungsorientierten Unternehmens entfällt auf die Personalkosten. Deshalb ist es sinnvoll, in dieses Potential zu investieren. Genau hier liegt die Bedeutung der Personalentwicklung.

Wo der Wandel sich beschleunigt, die wirtschaftlichen Spielräume immer enger werden und ein Mangel an leistungsstarken Mitarbeitenden zu erwarten ist, hängt die Leistungsfähigkeit eines Unternehmens entscheidend davon ab, wie mit dem Wirkungsgrad der »Ressource Mitarbeitende « umgegangen wird. Das Mitarbeiterpotential ist ein Betriebsvermögen, das in keiner Bilanz auftaucht, für den Wert eines Unternehmens aber von entscheidender Bedeutung ist. Je besser die Mitarbeitenden ausgebildet und ihren Fähigkeiten und Begabungen entsprechend in das Unternehmen eingebunden sind,
Aufgabe der Personalentwicklung ist es also, Mitarbeiterpotentiale zu erkennen und zu fördern, auf Berufsbiographien aktiv Einfluss nehmen, für derzeitige Aufgaben zu qualifizieren und für künftige Herausforderungen vorzubereiten. Mitarbeitende wollen vorhandenen Fähigkeiten und Neigungen in den Arbeitsprozess einbringen. Sie wollen Entwicklungsmöglichkeiten haben und zielorientiert wirken.

\section{Vierter Erfolgsfaktor: Das Personalmarketing}

Ein gelungenes Personalmarketing schafft die Voraussetzungen für eine langfristige Absicherung eines Unternehmens mit qualifizierten und motivierten Mitarbeitern. Die richtigen Talente auf das eigene Unternehmen aufmerksam zu machen und es an sich zu binden, das ist derzeit die Herausforderung für das Management.

Die demographische Entwicklung zwingt auch sozialwirtschaftliche Un- ternehmungen dazu, sich in Bezug auf Mitarbeitendengewinnung professionell aufzustellen. Dazu gehört eine strategische Personalbedarfsplanung mit den entsprechenden Anforderungsprofilen für die Mitarbeitenden.

Bewerbungs- und Auswahlverfahren konzentrieren sich primär auf die Mitarbeitergewinnung. In transparenten und glaubwürdigen Verfahren entscheiden Mitarbeitende und Unternehmen, ob eine »Partnerschaft zur Erreichung von Unternehmenszielen « zu realisieren ist. Schnell spricht sich herum, ob sich ein Unternehmen positiv als Arbeitgeber positioniert hat. Auf Neudeutsch: »Employer Branding! «

\section{Fünfter Erfolgsfaktor: Das betriebliche Gesundheitsmanagement}

Die demografische Entwicklung kann nahezu sicher vorhergesagt werden. Bis zum Jahr 2020 wird es mehr über 50-jährige als unter 30-jährige Erwerbstätige geben. 50-jährige Mitarbeitende haben heute noch 17 Berufsjahre vor sich.

Die Unternehmen sind also gezwungen, die Leistungsbereitschaft und Leistungsfähigkeit ihres Personals über einen viel längeren Zeitraum zu erhalten. Das bedeutet für die Unternehmensleitung ein verstärktes Engagement in die Gesundheit ihrer Mitarbeitenden. In Unternehmen, in denen die "Kunden « oft Menschen mit erheblichen Beeinträchtigungen und Verhaltensauffälligkeiten sind, spielt neben den physischen Belastungen die psychische Beeinträchtigung der Gesundheit eine wesentliche Rolle. Arbeitsbedingungen und Arbeit-

\section{Wohlfahrtsverband}

Caritas

Diakonie

Rotes Kreuz

Paritätischer Wohlfahrtsverband

Arbeiterwohlfahrt
Stand

31.12 .2008

1.1.2008

31.12.2008

132.000

1.1.2008

31.12 .2008

»Die Freie Wohlfahrtspflege stellt die größten Arbeitgeber in Deutschland und ist in zukunftsrelevanten Märkten aktiv. Zu diesem Ergebnis kommt eine Studie der Deutschen Bank aus dem letzten Jahr.

Insgesamt beschäftigte sie 2008 1,54 Mio. Menschen. Seit 1970 hat sich die Beschäftigung in etwa vervierfacht. Dennoch wird diese Institution im aktuellen Wirtschaftsleben kaum wahrgenommen.

Internet http://www.dbresearch.de/PROD/DBR_INTERNET_DE-PROD/PRODooo0000000264932.pdf 
sprozesse haben auf den Erhalt der Gesundheit der Mitarbeitenden Rücksicht zu nehmen.

Chefinnen und Chefs, die das ignorieren, zerschleißen mutwillig ihr Leistungspotential und wundern sich dann, dass die Krankheitsquote so hoch ist und niemand bei ihnen arbeiten will.

\section{Sechster Erfolgsfaktor: \\ Das Diversity Management}

Die Verschiedenheit der Mitarbeitenden " wird in den kommenden Jahren zwangsläufig zunehmen. Immer mehr Menschen unterschiedlicher $\mathrm{Na}$ tionen, Kulturen, Religionen werden die personelle Vielfalt der Unternehmen prägen. Über einen langen Zeitraum werden Unternehmen verstärkt auf die Erfahrung von älteren Mitarbeitenden zurückgreifen. Junge und alte Mitarbeitende werden gemeinsam ihre Potentiale zum Wohle des Erfolges bündeln müssen. Unterschiedliche Weltanschauungen und religiöse Orientierungen fördern kreative und Innovative Prozesse. Die Wertschätzung und Wertschöpfung der Vielfalt bei den Mitarbeitenden wird mit entscheidend dafür sein, wie erfolgreich ihr Unternehmen in der Zukunft ist.

Die Offenheit von Unternehmen gegenüber einer bunten, vielfältigen Mitarbeiterschaft wird ihnen im Wettbewerb um gute Mitarbeitende große Vorteile verschaffen. Eine Kultur der Toleranz macht sie zu einem attraktiven Arbeitgeber.

\section{Praxisgerechte Unterstützung.}

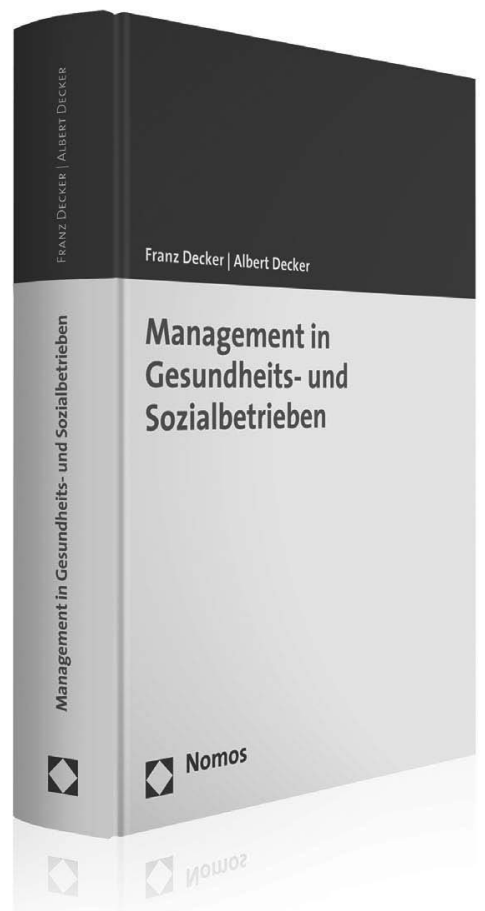

\author{
Management in Gesund- \\ heits- und Sozialbetrieben \\ Betriebswirtschaftliche Grund- \\ lagen für Führungskräfte und \\ Nachwuchs \\ Von Prof. Dr. Franz Decker und \\ Dr. Albert Decker \\ 2. Auflage 2008, 432 S., geb., 74,- $€$ \\ ISBN 978-3-8329-2990-9
}

Gesundheits-, Sozial-, Fitness- und Medizinbetriebe müssen wirtschaftlich gesteuert und gestaltet werden. Es wird immer wichtiger, Kunden überzeugend zu begleiten und Mitarbeiter professionell zu führen. Dieses moderne Betriebswirtschafts- und Management-Handbuch für Sozial- und Gesundheitsbetriebe gibt darauf praxisorientierte Antworten mit Fallbeispielen und Übungen.

"Das Werk von Franz und Albert Decker bietet in einer Zeit der sich ständig ändernden Imponderabilien einen Leitfaden für ein effizienzorientiertes Verhalten und Handeln in Gesundheits- und Sozialbetrieben. Es gehört zu jenen Werken, die im Sozialmanagement unerlässlich erscheinen."

Prof. Dr. Dr. habil. Peter Eisenmann, www.socialnet.de September 2009

"Mit Beispielen und Schaubildern verständlich präsentiert, bietet das Handbuch einen hervorragenden Grundriss. Es ist gleichermaßen für den Einstieg wie für die Vertiefung geeignet. Hier schreiben Praktiker, die wissen, worauf es ankommt."

Ronald Richter, Altenheim 3/o9

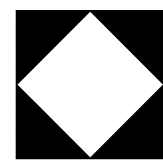

\section{Nomos}

Bitte bestellen Sie im Buchhandel oder versandkostenfrei unter www.nomos-shop.de 